\title{
HORACIO SANTIAGO-OTERO (1928-1997), AMIGO Y MAESTRO
}

\author{
José María Soto Rábanos \\ CSIC. Madrid
}

Era lunes aquel 26 de mayo de 1997. Como de costumbre, entre las nueve y las diez, esperaba su llamada para ir a tomar un café con leche y, al tiempo, comentar el fin de semana, lo bueno, lo menos bueno y hasta lo malo, arreglar un poco el mundo a nuestro modo radical/moderado/utópico y tomar nota de los quehaceres de la jornada, priorizando las tareas. Ese día pasaban ya bastantes minutos de las diez y aún no había llamado. Descolgué el teléfono para hacer yo la llamada, pero desistí; me dije que era lunes, día en el que a veces venía más tarde procedente de su casa de Carabaña, o que quizás habría tenido que ir de médicos, pues en los últimos cuatro/cinco meses frecuentaba a los cardiólogos que le habían revisado las pilas y la posición del «marcapasos», del que su corazón se ayudaba desde hacía más de 20 años para poder funcionar con cierta normalidad.

A las once, aproximadamente, sonó el teléfono; era Horacio. En un primer momento, pensé que me llamaba desde el despacho de su amigo Jesús Martín Tejedor, en el que disponía de una mesa de trabajo, donde podía desarrollar su tarea investigadora, siempre intensa, aun después de su jubilación a los sesenta y cinco años, en julio cumplido de 1994. Fueron solamente unos segundos, pues enseguida me aclaró que me llamaba desde casa, que había pasado un fin de semana pachucho y no se encontraba con fuerzas para ir a trabajar. Se había levantado con idea de ir, pero, ya se sabe, a veces, aunque el espíritu está pronto, la carne es débil y no permite el esfuerzo deseado. Le animé a reposar, minimizando a propósito la tarea del día; lo que no hagamos hoy, lo haremos al día siguiente, o cuando se pueda hacer, le dije.

Estábamos empeñados por esos días en los últimos toques de la preparación de una sección especial en el congreso quinquenal de la SIEPM (Société Internationale pour l'Étude de la Philosophie Médiévale, de la que era vicepresidente, a celebrar en Erfurt tres meses más tarde, entre el 25 y el 30 de agosto de 1997, sección que Horacio había diseñado con mimo y que preparaba con ilusión. Sentía gran preocupación por el congreso y por la sección a él encomendada. Dentro del tema general, Was ist Philosophie im Mittelalter?, Horacio había conseguido una sección dedicada a la filosofía medieval hispana de signo diferente a la filosofía escolástica, centrada en tres grandes autores, bajo el título: Filosofía y' filosofias en la edad media hispana. Raimundo Lulio (1235-1316), Arnaldo de Vilanova (ca.1238-1311), Ramón Sibiuda († 1436). La tarea se había encomendado a seis especialistas, dos por cada autor, de diferentes nacionalidades: 
dos españoles, dos alemanes, un portugués y un italiano; Horacio reservaba para si la coordinación y la exposición de conjunto. Disponíamos de los respectivos resúmenes, en lengua vernácula y en inglés.

Además, como de costumbre, coincidiendo con el congreso, la SIEPM celebraba Asamblea General, en la que, aparte la lectura y aprobación de los informes relativos a la actividad y estado económico de la sociedad, se debía proceder a la renovación de diversos miembros del Bureau. Horacio había luchado siempre por mejorar la representación latina en el Bureau frente al empuje noble, correoso y acaparador de centroeuropeos y norteamericanos. Poco a poco iba consiguiendo sus propósitos de equilibrio. Por de pronto, había conseguido unos años atrás, en el congreso de El Escorial (1991), que la lengua española fuera admitida como oficial, y por tanto utilizable, en la SIEPM.

No pudo observar el desarrollo del congreso de Erfurt, no pudo recoger los frutos de su esfuerzo, que fueron ciertamente en gran medida los que él hubiera deseado. Estoy seguro de que muchos miembros de la SIEPM pensaron en Horacio antes de tomar una decisión, de dar una opinión, de depositar su voto.

Estaba también a punto de llevar a cabo uno de sus proyectos preferidos: desplazarse a tierras portuguesas para impartir un curso de «mestrado» en la Universidad de Coimbra, al estilo del que había impartido anteriormente en la Universidad de Lisboa.

Apenas unas semanas antes, el 7 de mayo, había tenido lugar en el Salón de Actos de la Biblioteca Nacional la presentación de dos libros suyos: La cultura en la edad media hispana (1100-1470) y Estancia y predicación de Santiago apóstol en España según Roa Dávila, éste compartido con Klaus Reinhardt, con quien estuvo siempre unido por una gran amistad y por una colaboración profesional intensa.

A niveles personales, familiares, en ese mismo mes de mayo había gozado de una jornada de especial alegría para él. El 10 de mayo, sábado, su hijo Fernando, de diez años de edad recién cumplidos (el 26 de abril), había recibido la primera comunión en la iglesia de su colegio junto a otros niños. Aunque Horacio no fuera un «creyente» al estilo que se utiliza vulgarmente esta palabra, tenía grandes conocimientos por profesión y por vivencia de la cultura cristiana, y se sentía profundamente cristiano, más allá de los modos y reglas oficiales del catolicismo de ayer y de hoy, nucleado ese sentimiento en la persona de Jesucristo como figura central de la historia de la humanidad. El respeto por lo que representa la fe en Cristo, incluyendo a la generalidad de los «creyentes», aumentaba día a día en su mente y en su corazón. De ahí, creo yo, su especial alegría en ese día.

Se dijera que las cañas se tornaban lanzas para él. Se le veía animado, ilusionado en su próximo futuro, con nuevos bríos, renovado en su inquebrantable voluntad de trabajo. Pero ese día, lunes 26 de mayo, traicionó su perspectiva de futuro. Sin apenas avisar, suavemente, la muerte le vino a visitar; con dulzura, sin dolor, pero implacable. Acostado en su cama, leía el Bulletin de la SIEPM; en un momento, el libro resbaló de entre sus manos; y el sueño se apoderó de él para siempre. Su mujer, María Fernanda Cardama (Nana) lo llamó para comer algo suave que previa consulta le había preparado; no obtuvo respuesta. Horacio yacía inerme con el libro a su lado. Sus vecinos y buenos amigos Julio y Luis se ocuparon inmediatamente del papeleo post mortem. Tuvo una muerte dulce sí, pero muerte al fin. Descanse en paz. 
Los que hemos tenido la suerte de vivir el trabajo al lado de Horacio y de contar con su amistad sabemos bien de su entrega ilusionada a la investigación y del valor de sus sentimientos de amigo. No llegó a dar de sí todo cuanto tenía dentro, porque la muerte nos lo llevó cuando se hallaba en un momento excelente de realizaciones y proyectos, con buen reconocimiento de sus méritos en los ámbitos científicos (Académico de Mérito de la Academia Portuguesa da História, Vicepresidente de la SIEPM «Société Internationale pour l'Étude de la Philosophie Médiévale», premio Laranjo Coelho concedido por la Academia Portuguesa da Historia); pero Horacio deja un legado científico altamente meritorio como investigador y como promotor de los estudios de investigación científica y técnica en el área de las humanidades.

Horacio Santiago-Otero había nacido en Donadillo, un pequeño pueblo de la provincia de Zamora, el 20 de julio de 1928; estaba, pues, próximo a cumplir 69 años de vida.

La formación académica de Horacio Santiago-Otero se centró en el estudio y conocimiento de la teología, de la filosofía y de la historia en general, y su especialización científica se orientó hacia la historia del pensamiento religioso y filosófico en la época medieval, con atención a la búsqueda de documentos (fundamentalmente inéditos), a la transcripción y al estudio de textos manuscritos, y al análisis histórico.

Tras las primeras letras en su localidad natal, inició su formación humanística, filosófica y teológica en el Seminario Diocesano de Astorga, y la continuó en la Universidad Pontificia de Salamanca, donde dio los primeros pasos de investigador con la elaboración de un estudio histórico sobre los alumbrados del reino de Toledo, trabajo previo a la obtención del grado de Licenciado en Teología por la Universidad Pontificia de Salamanca en 1953. En esa misma universidad realizó el curso del doctorado.

Entre 1954 y 1967 amplió su formación académica y elaboró la tesis doctoral. Para ambos fines, hubo de frecuentar aulas y bibliotecas. Asistió a cursos varios de historia de la filosofía y la teología en París (Universidad Católica y la Sorbona) y de ciencias auxiliares de la historia (biblioteconomía, paleografía, diplomática) en Roma (Biblioteca Apostólica Vaticana).

Contó con el apoyo y la dirección de prestigiosos maestros como Paul Henry y Jean Châtillon, de la Universidad Católica; Vladimir Jankélévitch, Jean Wahl, Henri Gouhier, Maurice de Gandillac, de la Facultad de Filosofía de la Sorbona; Paul Vignaux, André Combes, Henri Christian Puesch, Jean Orcibal y Jean Jolivet, de l'École pratique des hautes études (La Sorbona); Henri Irenée Marrou, de la Facultad de Historia (La Sorbona); Marie Thérèse d"Alverny (Bibliothèque Nationale), entre otros. Realizó varias visitas a la Abbaye du Mont César (Lovaina) para entrevistarse con los afamados medievalistas Hildebrand Bascour y Odon Lottin.

Las bibliotecas parisinas, con sus ricos fondos inéditos e impresos, constituyeron el marco donde fundamentalmente trabajó durante esos años, en especial la «Nationale», la «Mazarine» y la «de l'Arsenal».

Durante esa misma época, realizó una ardua y amplia labor investigadora, consultando fondos inéditos relativos a documentos teológicos y filosóficos medievales en numerosas bibliotecas de Europa: Arras, Avranches, Bamberg, Bruges, Bruxelles, Cambridge, Firenze, Klosterneuburg, London, Madrid, München, Oxford, Saint-Omer, Salzburg, Stuttgart, Wien.

Consultó, además, documentos inéditos de otras bibliotecas a través de los servicios del Institut de Recherche et d'Histoire des Textes (CNRS, París) y del Grabmann Institut (Universidad de Munich). 
Los cursos 1963-1967 continuó su actividad investigadora en diversos centros romanos, como miembro del Instituto de Historia Eclesiástica adscrito a la Iglesia Nacional Española de Santiago y Montserrat. Ello le permitió utilizar los valiosos recursos que ofrecen las bibliotecas romanas, en especial la Vaticana, donde pudo completar las investigaciones relativas al tema de la tesis doctoral, a la vez que iniciaba y desarrollaba otras referentes a temas de historia de la teología y de la filosofía, de historia del pensamiento y de las instituciones medievales.

Trabajó asimismo en otras bibliotecas romanas bien conocidas para el estudioso medievalista: Angelica, Casanatense, Nazionale Centrale Vittorio Emanuele II, Vallicelliana, y en otras varias de centros romanos universitarios y culturales.

En 1967 defendió, en la Universidad Católica de París, su tesis doctoral sobre el conocimiento de Cristo en los teólogos del siglo XII, obteniendo la puntuación máxima.

Entre 1967 y 1972, se ejercitó como profesor de Teología y de Historia de temas teológicos y filosóficos medievales en el Instituto Central de Cultura Religiosa Superior de Madrid, en la Facultad de Teología del Norte de España (con sede en Burgos) y en la Universidad Pontificia de Salamanca. En realidad, Horacio se había iniciado en el profesorado en el curso 1961-1962, en el Seminario Mayor de Astorga, donde explicó a lo largo de dos años académicos varios tratados teológicos: sobre el misterio de Dios, introducción a la teología, cuestiones trinitarias, cuestiones cristológicas, teología de la gracia, al tiempo que se hacía cargo de la biblioteca del Seminario.

En el Instituto Central de Cultura Religiosa Superior de Madrid ocupó en los cursos 19671972 la cátedra de teología de la gracia y de 1969 a 1972 la de historia de la teología, con especial atención a la época medieval.

En el Estudio Teológico de Madrid explicó los tratados de cristología y de mariología (19671968) y el tratado sobre el misterio de Dios (1967-1968, 1970-1972), a la vez que dirigió un curso sobre la cristología en Teilhard de Chardin (1968-1969).

En la Facultad de Teología del Norte de España (con sede en Burgos) fue profesor de cristología (1968-1970) y de antropología teológica (1968-1969). Dirigió además un seminario sobre títulos cristológicos en los padres de la iglesia (1969-1970).

En la Universidad Pontificia de Salamanca fue profesor, en la Facultad de Teología, de teoría general de los sacramentos y de historia de la teología medieval durante los cursos 1969-1972, y dirigió un seminario sobre el conocimiento religioso en la teología francesa de los últimos años: de Blondel a Teilhard de Chardin (1971-1972). En abril de 1976 dictó un cursillo sobre introducción a santo Tomás de Aquino. En la Facultad de Filosofía de dicha universidad salmantina explicó anualmente, entre 1973 y 1977, un cursillo de 15 a 20 lecciones sobre diversos temas en relación con la historia de la filosofía medieval; y en noviembre de 1976 dictó seis ponencias sobre los grandes maestros del siglo XII.

Fuera de estos paréntesis docentes, se puede afirmar que, desde 1968 hasta su inesperada muerte el 26 de mayo de 1997, Horacio fue un investigador dedicado al CSIC. Fueron 29 años de dedicación apasionada a la investigación en la institución que él amaba por encima de los avatares y de las circunstancias políticas, profesionales y personales que le tocó vivir: 4 como becario postdoctoral y doctor contratado, 21 como investigador en plantilla, 4 más de forma voluntaria después de su jubilación en julio de 1993. 
En 1968 comenzó a trabajar en el CSIC (Consejo Superior de Investigaciones Científicas) en calidad de becario postdoctoral.

En sendas oposiciones, ganó las plazas de Colaborador (1972) y de Investigador Científico (1979) del CSIC dentro del Instituto Francisco Suárez, en la línea de investigación de la historia de la teología, de la filosofía y de la actividad cultural en la edad media, especialmente de la cultura medieval hispana.

Junto a su apreciable labor personal investigadora, que se traduce en una abundante bibliografía, y aparte los cargos que desempeñó en el CSIC, Horacio Santiago-Otero desplegó una gran actividad procientífica.

Durante los últimos años de la dictadura y en los años de la transición buscó siempre elevar el nivel de la investigación y de las relaciones humanas entre el personal dedicado directa $o$ indirectamente a la investigación, así como situar la actividad científica e investigadora en el plano de las libertades y de las responsabilidades políticas institucionales que son propias de la democracia. En este sentido, es de recordar la fundación del club «Marco Aurelio», presidido por el profesor José Luis López Aranguren, y del que Horacio Santiago fue secretario general y alma mater durante el tiempo que duró, corto pero intenso en lecciones y en debates de actualidad.

Participó asimismo durante varios años en trabajos de política científica con grupos diferentes e intervino en la elaboración de documentos destinados a analizar la problemática de la investigación en España (especialmente a nivel de las Humanidades) y la situación del CSIC, poniendo de manifiesto su interés y preocupación por los problemas de política científica, y su conocimiento de los problemas con ella relacionados a nivel del CSIC y a nivel nacional. En este sentido, se puede recordar su participación en el seminario sobre «El Consejo Superior de Investigaciones Científicas en el Estado de las Autonomías», que se celebró en Madrid los días 14, 21 y 28 de junio de 1988, y en el que Horacio tuvo un protagonismo muy destacado.

Con la vista siempre hacia delante, Horacio se ocupó hasta donde pudo, con la disponibilidad y la ilusión en él habituales, en tareas procientíficas, mediante la organización de congresos y reuniones de estudio, la consecución y dirección de proyectos de investigación, la orientación de becarios y de investigadores jóvenes, así como la participación en sociedades y asociaciones científicas nacionales e internacionales en calidad de miembro directivo. A lo dicho cabe añadir la coordinación y edición de misceláneas y su labor de editor y coeditor en varias series y colecciones.

De sus actividades en favor de la investigación humanística, quiero destacar aquí dos aspectos que le preocupaban más y más cada día. De una parte, acrecentar los estudios sobre la historia cultural hispana bajomedieval, campo de trabajo poco atendido por los medievalistas hispanos, y difundirlos en el ámbito científico de la comunidad europea e internacional; de otra parte, extender los contactos culturales entre España y Portugal, en especial los relativos a la investigación humanística en la edad media.

Su actividad en el CSIC no le impidió seguir investigando en bibliotecas y archivos en acopio de documentación. La tarea realizada, muy particularmente en las ciudades de París, Roma, Munich y Madrid, prueba su preocupación intensa por los temas relativos a la historia del pensamiento religioso y filosófico y a la historia de las instituciones. Se ocupó de la búsqueda de nuevos datos para el «fichero de autores hispanos», e inició uno nuevo de autores hispano cristianos (traductores, teó- 
logos, filósofos, juristas, etc.) de la época medieval, que sirvió de base a un proyecto titulado: «Nomenclátor de autores medievales hispanos».

Dedicó particular atención al estudio del teólogo salmantino Juan de Segovia y colaboró en la transcripción de dos importantes repetitiones suyas inéditas, con vistas a la publicación de las mismas: la Repetitio de superioritate et excellentia supremae potestatis ecclesiasticae et spiritualis ad regiam et temporalem, según el manuscrito de la biblioteca de Santa Cruz de Valladolid, n. ${ }^{\circ} 65$, fol. 130r-165r (leída por Juan de Segovia en Salamanca el 6 de julio de 1426) y la Repetitio de fide catholica facta per Johannem Alfonsi de Segovia in theologia magistrum, según el manuscrito de la biblioteca capitular de Córdoba, n. ${ }^{\circ}$ 128, fol. 182vb-193rb (leída por Juan de Segovia en Salamanca el día 22 de mayo de 1427). El primero de estos dos escritos es de particular importancia para la historia de las ideas, ya que a través de él se demuestra que Juan de Segovia no era conciliarista por esas fechas, y que probablemente no lo fueran ninguno de los maestros salmantinos de entonces, no obstante alguna opinión reciente en sentido contrario. Otro autor al que Horacio dedicó una atención preferente fue Pedro Martímez de Osma, como puede apreciarse por sus publicaciones sobre el mismo.

Sus estancias, a veces de semanas a veces de meses, en países europeos y americanos, así como su asistencia a coloquios y congresos, le permitieron establecer contactos con estudiosos destacados en el campo de la cultura e investigación histórica y con centros especializados de diversos países: Centre Européen de Recherches sur les Congrégations et Ordres Religieux (SaintEtienne); Cátedra de Historia de los Dogmas e Institut für Cusanus-Forschung (Trier); Instituto de Historia Eclesiástica (Roma); Société Internationale pour l'Étude de la Philosophie Médiévale (Louvain-La-Neuve); Institute of Medieval Canon Law y Department of Spanish and Portuguese (Berkeley-Cálifornia-USA); Pontifical Institute of Medieval Studies (Toronto); Cátedra de Historia da Filosofía y Academia Portuguesa da História (Lisboa); Centro de Estudios Dominicanos (Porto); Arquivo da Universidade e Instituto de História e Teoria das Ideias (Coimbra); Cátedra de Historia de las Instituciones Canónicas (Siena); Raimundus Lullus Institut (Freiburg-Alemania); Hispanic Society, (New York).

En diferentes ocasiones gozó de becas y ayudas destinadas sucesivamente a la especialización y a la investigación en el extranjero y en España, que hicieron posibles sus estancias de estudio y consulta: de Relaciones Culturales Francesas (París, verano de 1954); del Comité Catholique des Amitiés Françaises dans le Monde v (París, 1955-1956); del Patronato del Gobierno Francés (París, 1955-1959); del Ministerio de Educación y Ciencia (París 1956-1958); del Ministerio de Asuntos Exteriores (Iglesia Nacional Española de Santiago y Montserrat, Roma 1964-1967); del Consejo Superior de Investigaciones Cientificcas (Instituto Francisco Suárez, Madrid 1968-1970); de la Deutsche Forschungsgemeinschaft (bibliotecas de Munich y Trier, varios veranos en el mes de agosto); del Consejo Superior de Investigaciones Científicas (contrato de trabajo para el Instituto Francisco Suárez, 1970-1972); del Consiglio Nazionale delle Ricerche (Roma, septiembre 1978); del Comité Conjunto Hispano-Norteamericano para Asuntos Educativos y Culturales (Institute of Medieval Canon Law de la Universidad de Berkeley, California-USA, julio-agosto de 1980).

Las publicaciones de Horacio Santiago-Otero se refieren en su gran mayoría a temas del pensamiento teológico, filosófico y religioso, de historia del pensamiento en general y de historia de 
Ias instituciones. Son fundamentalmente comentarios y también repertorios, aportación e indicación de fuentes. Selecciono las aportaciones más significativas por orden cronológico. ${ }^{1}$

- «El conocimiento de Cristo según las enseñanzas de Anselmo de Laon y de su escuela», Salmanticensis, 13 (1966), pp. 61-79.

- «Manuscritos de teológos medievales españoles en el Fondo Reginense Latino de la Biblioteca Vaticana (siglos XII-XV)», RHCEE, 1 (Salamanca 1967), pp. 353-376.

- «La ciencia beatífica de Cristo hombre según Pedro Abelardo y su escuela», Miscellanea André Combes, 2 (Roma 1967), pp. 95-147.

- «Gualterio de Mortagne ( $\dagger$ 1147) y las controversias cristológicas del siglo XII», RET, 27 (1967), pp. 271-283.

- «Juan de Segovia. Manuscritos de sus obras en la Biblioteca Nacional de Viena y en la Staatsbibliothek de Munich», RET, 29 (1969), pp. 167-179.

- El conocimiento de Cristo en cuanto hombre en la teología de la primera mitad del siglo XII; de la exclusiva ciencia divina del alma de Cristo (escuela de Laon) a los primeros interrogantes sobre su saber experimental (corriente monástica), Pamplona, Ediciones Universidad de Navarra 1970, 282 pp.

- Miscelánea Manuel Cuervo López; homenaje de antiguos alumnos, Salamanca 1970, 574 pp.; coordinador y editor de la obra; autor de las pp. 7-16 (Presentación de la obra y Bibliografía de Manuel Cuervo López) y 101-113, bajo el título: «La ciencia humilde de Jesús en Ricardo de San Víctor (+1173)».

- «Pablo de Santa María. Manuscritos de sus obras en la Staatsbibliothek de Munich», RET, 30 (1970), pp. 81-92.

- «Juan de Segovia. Algunos manuscritos de sus obras en la Biblioteca Vaticana», RET, 30 (1970), pp. 93-106.

- «Guido de Monte Roterio. Manuscritos de sus obras en la Staatsbibliothek de Munich», RET, 30 (1970), pp. 391-405.

- «Juan de Torquemada. Manuscritos de sus obras en la Staatsbibliothek de Munich», RET, 32 (1972), pp. 453-469.

- «Juan de Palomar. Manuscritos de sus obras en la Staatsbibliothek de Munich», RET, 33 (1973), pp. 47-57.

- Panorama actual de la teología española, Fundación Universitaria Española, Madrid 1974614 pp.; coordinador y editor de la obra; autor de las pp. 497-521, con el mismo título de la obra.

1 Siglas utilizadas:

AHDP: Arquivo Histórico Dominicano Português.

HEMP: Historia de España Menéndez Pidal.

HRT: Humanismo, Reforma y Teología.

RET: Revista Española de Teologia.

RHCEE: Repertorio de Historia de las Ciencias Eclesiásticas en España. 
- Miscelánea en honor de Juan Becerril y Antón-Miralles 1-2 Revista de Occidente, Madrid, 1974 XXV+667+605 pp.; coordinador y editor de la obra; autor de las pp. I. xiiixxi (Presentación de la obra y Biliografía de J. Becerril) y II. 571-581, bajo el título: «Acardo de San Víctor; un aspecto de su visión cristológica».

- «Juan de Palomar. Manuscritos de sus obras en la Biblioteca Vaticana», RET, 34 (1974), pp. 251-255.

- «Pedro Alfonso. Manuscritos de sus obras en la Staatsbibliothek de Munich», RET, 35 (1975), pp. 101-103.

- «Alfonso Buenhombre. Manuscritos de sus obras en la Staatsbibliothek de Munich», RET, 36 (1976), pp. 359-375.

- «El término teología en Pedro Abelardo», RET, 36 (1976), pp. 251-259; y Miscellanea Mediaevalia 13/2 Sprache und Erkenntnis im Mittelalter, Berlin-New York, 1981, pp. 881-889.

- «Manuscritos del Liber creaturarum de Ramón Sabunde», RET, 37 (1977), pp. 201-203.

- Antropología y teología, Madrid, CSIC, 1978, 152 pp.; coordinador-editor y coautor.

- Abelardo y Eloísa, Madrid, 1978, 18 pp.

- Siglo XII: los primeros intelectuales, Madrid, 1978, 18 pp.

- Siglo XII: origen de los centros intelectuales, Madrid, 1979, 16 pp.

- Humanismo y tecnología en el mundo actual; actas de las Jornadas de Investigación.

- Humanística, Madrid 11:16 de abril 1977, Madrid, CSIC, 1979, 194 pp.; coodinadoreditor.

- Simbolismo, sentido y realidad, Madrid, CSIC, 1979, 144 pp.; coordinador-editor.

- Investigación y democracia, Madrid, 1980, 16 pp.

- «Guido de Monte Roterio y el Manipulus curatorum», Proceedings of the Fifth International Congress of Medieval Canon Law, Salamanca, 21-27 de septiembre de 1976, Città del Vaticano 1980, pp. 259-265.

- «Tres sermones de Pedro Martínez de Osma», Celtiberia, 30 (1980), pp. 67-87. Colaboración con Klaus Reinhardt y Andrés Barcala Muñoz.

- Los cardenales Vidal i Barraquer, Segura y Pla i Daniel: tres opciones en el contexto Iglesia-Estado, Madrid, 1.981, $16 \mathrm{pp}$.

- La cátedra y el púlpito frente a frente: Pedro Abelardo y san Bernardo, Madrid, 1983, $24 \mathrm{pp}$.

- «Esse et habere en Hugo de San Víctor», RET, 43 (1983), pp. 175-179; L'homme et son univers au moyen âge 1 (Louvain-La-Neuve 1986), pp. 427-431.

- Las instituciones jurídicas en algunas escuelas medievales de la Península Ibérica (HRT, 56; Estudios, 18; CSIC, Madrid 1984) 24 pp. Colaboración con M ${ }^{a}$ Teresa Cardó.

- Pedro Abelardo y la «licentia docendi», Madrid, 1984, 22 pp.

- Críticas y aportación de Pedro Martínez de Osma (+1480) al método teológico (HRT 57; 
- Estudios 19; CSIC, Madrid 1984) 46 pp. Colaboración con K. Reinhardt.

- «La escuela catedral de Burgo de Osma en tiempos de santo Domingo de Guzmán», AHDP, 3/1 (Porto 1984), pp. 119-122.

- «Los Dialogi de Pedro Alfonso: tradición manuscrita e impresa», Azafea, 1 (1985), pp. 33-43. Colaboración con K. Reinhardt.

- «El Sacramental de Clemente Sánchez de Valderas», Monumenta iuris canonici, series C: subsidia, vol.7 (Città del Vaticano 1985), pp. 153-161.

- Biblioteca bíblica ibérica medieval (Medievalia et Humanistica 1; Nomenclátor de Autores Medievales Hispanos 1; CSIC, Madrid 1986) XXVIII+384 pp. Colaboración con K. Reinhardt.

- «Guido de Monte Roterio. Nuevos manuscritus del Manipulus curatorum», Homenaje a - Pedro Sáinz Rodríguez, 1 (Madrid 1986), pp. 15-20.

- Pedro Martínez de Osma y el método teológico: edición de algunos escritos inéditos (Medievalia et Humanistica, 2; CSIC, Madrid 1987) 202 p̣p. Colaboración con K. Reinhardt.

- Manuscritos de autores medievales hispanos I (Medievalia et Humanistica, 3; CSIC, Madrid 1987) 204 pp.

— «La formación de los clérigos leoneses en el siglo XII», Isidoriana, 1 (León 1987), pp. 177-191.

- «Virgilio Cordobés y las escuelas medievales», Athlon. Satvra grammatica in honorem Francisci R. Adrados, 2 (Madrid 1987), pp. 817-822.

- Fe y cultura en la edad media (Medievalia et Humanistica 4; CSIC, Madrid 1988) $306 \mathrm{pp}$.

- «Comentaristas bíblicos de los siglos XII y XIII», Coloquio sobre circulación de códices y escritos entre Europa y la Península Ibérica en los siglos VIII-XIII (Santiago de Compostela 1988), pp. 193-208; en colaboración con K. Reinhardt.

- «La Biblia en maestros dominicos de la Península Ibérica durante la edad media», Actas do III encontro sobre história dominicana II (AHDP, IV/2; Porto 1989), pp. 277-289; en colaboración con Klaus Reinhardt.

— «Transmission de savoirs à Tolède à l'époque de la reconquête», Tolède (1085-1985). Des traductions médiévales au mithe littéraire. Actes du colloque de Mulhouse décembre 1985 (Paris 1989), pp. 41-55.

- «The Libro declarante: an anonymous work in the anti-jewish polemic in Spain», Tenth World Congress of Jewish Studies, division B, vol. II (Jerusalem 1990) 77-82.

- «Instituciones y espiritualidad medievales en la Península Ibérica: escuelas y bibliotecas capitulares», $I^{a s}$ Jornadas Académicas de História da Espanha e de Portugal, (Lisboa 1990), pp. 27-42.

- El Camino de Santiago, la hospitalidad monástica y las peregrinaciones (Valladolid, Junta de Castilla y León 1992) 394 pp.; coordinador-editor. 
- «Juan Roa Dávila (1552-ca.1630) y las controversias sobre la venida y predicación de Santiago en España», El Camino de Santiago, la hospitalidad monástica y las peregrinaciones, pp. 17-26.

- «As controversias sobre a vinda e pregação do Apóstolo Santiago em Espanha, segundo Roa Dávila (1552-ca.1630)»

- I Congresso Internacional dos Caminhos Portugueses de Santiago de Compostela, (Lisboa 1992), pp. 42-52; en colaboración con Klaus Reinhardt.

- «Escritos de polémica antijudía en lengua vernácula», Mediaevalia, 2 (1993), pp. 185195; en colaboración con Klaus Reinhardt.

- Diálogo filosófico-religioso entre cristianismo, judaísmo e islamismo durante la edad media en la Península Ibérica (Turnhout, Brepols 1994) XI-508 pp.; coordinador-editor.

- «La sistematización del saber y su transmisión entre la minoría culta: escuelas, universidades, escritura, libro y bibliotecas»,HEMP, 16 (Madrid 1994), pp. 791-828; en colaboración con José María Soto Rábanos.

- «Los saberes y su transmisión en la Península Ibérica (1200-1470)», Medievalismo. Boletín de la Sociedad Española de Estudios Medievales, 5 (1995), pp. 213-256; en colaboración con José María Soto Rábanos.

- «Juan Gil de Zamora: comentarios a la Biblia», Amar, sentir e viver a história. Estudos de homenagem a Joaquim Veríssimo Serrão, 1 (Lisboa 1995), pp. 435-443.

- «Dualidad religioso-cultural del Camino de Santiago», Actas del Congreso de Estudios Jacobeos, (Santiago de Compostela 1995), pp. 645-650.

- La cultura en la edad media hispana (1100-1470), (Lisboa, Colibri 1996) 156 pp.

- Estancia y predicación de Santiago Apóstol en España según Roa Dávila, Madrid, CSIC, 1996108 pp.; en colaboración con Klaus Reinhardt.

- «Fundamentación filosófica del pensamiento antoniano. Perspectiva ética», Congresso Internacional Pensamento e Testemunho. $8^{\circ}$ Centenário do Nascimento de Santo António. Actas, 1 (Braga 1996), pp. 99-110.

- «Cartas del Profesor Caeiro», In memoriam do Professor Doutor Francisco da Gama Caeiro, (Lisşboa 1996), pp. 101-118.

- «Pedro Alfonso. Obras y bibliografía», Estudios sobre Pedro Alfonso de Huesca (Huesca 1996), pp. 19-44; en colaboración con Klaus Reinhardt.

- «La mística hispana en los siglos XVI y XVII», Mística, pensamiento y cultura. Tercer centenario de la muerte de Miguel de Molinos, (Zaragoza 1996), pp. 205-224.

- «Los estudiós generales en Hispania y la fundación del Estudio de Alcalá de Henares», L. Jiménez (coord.) La universidad complutense cisneriana, (Madrid 1996), pp. 45-61.

- «Presencia e intervención de conversos en la génesis del iluminismo hispano (comienzos del siglo XVI)», Actas dos $2{ }^{\text {os }}$ Cursos de Verão de Cascais (17 a 22 de julho de 1995), 1 (Cascais 1996), pp. 195-210. 
- «El derecho canónico en el contexto del Libro de buen amor», Estudios de frontera. Alcalá la Real y el Arcipreste de Hita. Actas (Jaén 1996) 615-631.

- «La escuela catedralicia de Santiago de Compostela (siglos XI-XIII)», Miscelánea Joaquim Veríssimo Serrão, (Lisboa 1996).

José María Soto Rábanos

C.S.I.C.

Duque de Medinaceli, 6 .

28014. Madrid 